\title{
RELAÇÃO DOS PROFESSORES QUE EXERCERAM SUAS ATIVIDADES NO DEPARTAMENTO DE HISTÓRIA/UFPR, 1959-2009
}

\author{
Members of staff of the Department of \\ History/UFPR between 1959-2009
}

PROFESSORES DO CURSO DE HISTÓRIA E GEOGRAFIA, FACULDADE DE FILOSOFIA, CIÊNCIAS E LETRAS, UNIVERSIDADE DO PARANÁ, FUNDADORES DO DEPARTAMENTO DE HISTÓRIA, POR ANO DE INGRESSO*

Bento Munhoz da Rocha Netto 1938

Brasil Pinheiro Machado 1938

Homero Batista de Barros 1938

Altiva Pilatti Balhana 1951

Cecília Maria Westphalen 1951

Neusa de Castro Guimarães 1958

PROFESSORES DO DEPARTAMENTO DE HISTÓRIA DA UFPR, 1959-2009

Odah Regina Guimarães Costa 1962 (Instrutor Voluntário 1960) Jayme Antonio Cardoso 1964

Ruy Christovam Wachowicz $\quad 1966$

Oksana Olga Boruszenko 1967

Beatriz Pellizzetti 1968

Mitiko Okazaki 1969

Carlos Roberto Antunes dos Santos 1970 (Instrutor Voluntário 1967) Helena Isabel Mueller 1970

* Os professores das Cátedras de História da Filosofia, História da Educação e História das Doutrinas Econômicas não referendaram a proposta final do Departamento de História. 
Raquel Costa da Rocha Loures

Sergio Odilon Nadalin

Ana Maria de Oliveira Burmester

Elvira Mari Kubo

Márcia de Campos Graf

Maria Ignês Mancini de Boni

Mariza Budant Schaaff

Jair Mequelusse

Joseph Patrick McGovern

Manoel Henrique Roskamp

Alcina Maria de Lara Cardoso

Beatriz Teixeira de Melo Miranda

Maria Ivone Bergamini

Regina Rottemberg Gouvêa

Aída Mansani Lavalle

Lando Rogério Kroetz

Marília Souza do Valle

Etelvina Maria de Castro Trindade

Carlos Alberto Balhana

Judite Maria Barboza Trindade

Márcia Terezinha Daledone Siqueira

Marionilde Dias Brepohl de Magalhães

Francisco Moraes Paz

Euclides Marchi

Ronald Raminelli

Dennison de Oliveira

Magnus Roberto de Mello Pereira

Maria Auxiliadora Schmidt

Maria Luiza Andreazza

Ana Paula Vosne Martins

José Roberto Braga Portella

Luiz Geraldo Santos da Silva

Cacilda da Silva Machado

Luiz Carlos Ribeiro

Marcos Napolitano de Eugenio

Carlos Alberto Medeiros Lima

Renato Lopes Leite
1970

1970 (Instrutor Voluntário 1967)

1971

1971

1971

1971

1971

1972

1972

1972

1973

1973

1973

1974

1978

1978

1978

1980

1981

1982

1982

1986

1990

1990

1990

1991

1991

1991

1991

1993

1993

1993

1994

1994

1994

1996

1996 
Renan Frighetto 1997

Antonio César de Almeida Santos 1998

Fátima Regina Fernandes Frighetto 1998

Helenice Rodrigues 1998

Renata Senna Garraffoni 2004

Andréa Carla Doré 2005 (PRODOC 2003)

Marcella Lopes Guimarães 2006

Roseli Terezinha Boschilia 2006 (Professora Subst. 2005)

Joseli Maria Nunes Mendonça 2008

Martha Daisson Hameister 2008

PROFESSORES DO DEPARTAMENTO DE HISTÓRIA, PROGRAMA DE ABSORÇÃO TEMPORÁRIA DE DOUTORES (PRODOC/CAPES).

Andréa Carla Doré 2003

Luiz Felipe Silvério Lima 2006

\section{PROFESSORES SUBSTITUTOS DO DEPARTAMENTO DE HISTÓRIA}

Maurício Elvis Schneider 1995

Sérgio Aguilar Silva 1996

Cássio da Silva Fernandes 2003

Sérgio Feldman 2003

Ana Paula Peters 2005

Rafael Rosa Hagemeyer 2005

Roseli Terezinha Boschilia 2005

Aruanã Antonio dos Passos 2006

Eva Lúcia Gavron 2006

Liz Andréa Dalfré 2006

Rafael Faraco Benthien 2006

José Josberto Montenegro Sousa 2007

Luiz Adriano Gonçalves Borges 2008 